\title{
Post-Operative Outcomes of Radiological and Histopathological Findings after Hydroxyapatite and Allogenic Bone Graft among Jaw Cyst Patients: A Randomized Clinical Trial
}

\author{
Showkat Mamun ${ }^{1}$, Ismat Ara Haider' ${ }^{2}$ Nasiruddin ${ }^{3}$, Tarin Rahman ${ }^{4}$, \\ ATM Tarifuzzaman Rubel ${ }^{5}$, Farah Diba ${ }^{6}$
}

\begin{abstract}
${ }^{1}$ Assistant Professor, Department of Oral and Maxillofacial Surgery, Dhaka Dental College and Hospital, Dhaka, Bangladesh; ${ }^{2}$ Professor and Head, Department of Oral and Maxillofacial Surgery, Dhaka Dental College and Hospital, Dhaka, Bangladesh; ${ }^{3}$ Professor and Head, Department of Oral and Maxillofacial Surgery, Shaheed Suhrawardy Medical College and Hospital, Dhaka, Bangladesh; ${ }^{4}$ Associate Professor, Department of Oral and Maxillofacial Surgery, Dhaka Dental College and Hospital, Dhaka, Bangladesh; ${ }^{5}$ Assistant Professor, Department of Oral and Maxillofacial Surgery, Dhaka Dental College and Hospital, Dhaka, Bangladesh; ${ }^{6}$ Junior Consultant (Paediatrics), Upazilla Health complex, Keranigonj, Dhaka, Bangladesh
\end{abstract}

[Received: 12 August 2019; Accepted: 20 October 2019; Published: 1 January 2020]

\begin{abstract}
Background: Post-surgical outcomes of jaw cyst is very crucial. Objective: The purpose of the present study was to observe the radiological and histological finings as postoperative outcomes after hydroxyapatite and allogenic bone graft among jaw cyst patients. Methodology: This single centre, parallel, randomized clinical trial was conducted in the Department of Oral and Maxillofacial Surgery at Bangabandhu Sheikh Mujib Medical University, Dhaka, Bangladesh from July 2006 to June 2008 for a period of two (02) years. Patients presented with radicular and dentigerous cyst with the size of the lesion between $2 \mathrm{~cm}$ to $7 \mathrm{~cm}$. in diameter and relatively non infected cystic lesion were selected as study population. The study population were divided into two groups designed as group A treated with hydroxyapatite alloplastic material and group B treated with allogenic-bone graft after enucleation of the cystic lesion. The standard surgical procedure was to be performed meticulously in sterile environment. Post-operative radiograph were taken after one month, three months and six months. Histopathological examination was done after six month of operation which was done in the department of Pathology, BSMMU. Scintigraphic examination was performed after 6 months of the operation to evaluate the viability of the bone graft in the graft site. Result: A total number of 28 patients were recruited for this study of which 14 patients were treated with hydroxyapatite crystals bone graft in group A and 14 patients were treated with allogenic bone graft in group B. The age of patients of both groups ranged between 14 and 58 years. In histopathological examination marked presence of cementing line, woven bone and osteoblast cell was found in patients who were grafted with hydroxyaptite and this had indicated good osteoblastic activity. Increased technetium-99m labeled diphosphonate uptake was found in the patient with allograft. There was a smaller lesion diameter of hydroxyapatite group in comparison with allogenic bone graft group which had indicated comparatively better healing than that of the hydroxyapatite group. Conclusion: In conclusion hydroxyapatite alloplastic material has better radiological and histopathological outcomes than allogenic bone graft after surgery of jaw cyst. [Journal of National Institute of Neurosciences Bangladesh, 2019;6(1): 48-53]
\end{abstract}

Keywords: Radio-Histological Findings; Post-Operative Outcomes; Hydroxyapatite; Allogenic Bone Graft; Jaw Cyst Patients; Randomized Clinical Trial

Correspondence: Dr. Showkat Mamun, Assistant Professor, Department of Oral and Maxillofacial Surgery, Dhaka Dental College and Hospital, Dhaka, Bangladesh; Email: mamunshowkat@gmail.com; Cell no.: +8801715025367

Conflict of interest: There is no financial conflict of interest relevant to this paper to disclose.

Funding agency: This research project was not funded by any group or any institution.

Contribution to authors: Mamun S, Haider IA, Nasiruddin contributed from the protocol preparation, data collection up to report writing. Manuscript writing was performed by Mamun S. Rahman T, Rubel ATMT, Diba F have revised the manuscript.

How to cite this article: Mamun S, Haider IA, Nasiruddin, Rahman T, Rubel ATMT, Diba F. Post-Operative Outcomes of Radiological and Histopathological Findings after Hydroxyapatite and Allogenic Bone Graft among Jaw Cyst Patients: A Randomized Clinical Trial. J Natl Inst Neurosci Bangladesh, 2020;6(1): 48-53

Copyright: (C2020. Mamun et al. Published by Journal of National Institute of Neurosciences Bangladesh. This article is published under the Creative Commons CC BY-NC License (https://creativecommons.org/licenses/by-nc/4.0/). This license permits use, distribution and reproduction in any medium, provided the original work is properly cited, and is not used for commercial purposes. 


\section{Introduction}

Synthetic ceramics are readily available and are not either infectious or immunogenic potential ${ }^{1}$. These reduce patient morbidity significantly by decreasing the need for a second operative site. Calcium phosphates $(\mathrm{CaP})$ account for most ceramic-based bone graft substitutes currently available ${ }^{2}$. Hydroxyapatite (HA) is the most used polycrystalline calcium phosphate ceramic mineral as an artificial bone graft substitute ${ }^{3}$. It is biocompatible, highly resistant to stress forces, nontoxic and noncarcinogenic; furthermore, it has marked osteoconductive and osteoinductive properties that lead to the regeneration of lamellar bone within 4 to 10 months of implantation ${ }^{4}$. This characteristic is exploited in many surgical areas. It also has been used to coat other materials to increase their stability and osteointegration ${ }^{5}$.

The major drawbacks to the use of $\mathrm{CaP}$ ceramics are their adverse mechanical properties. These are brittle with low impact resistance, and have low tensile strength; consequently, these fail when used in a structural capacity ${ }^{4}$. The lack of viscous flow is a serious limitation for CPCs. Despite this problem, some researchers tried injecting CPCs in paste form; in addition it's tendency for granular migration and incomplete resorption has become a long-term problem ${ }^{6}$.

Allogenic bone is another attractive source of bone grafting material next to auto bone. Allogeneic bone, with variable biologic properties, is available in many preparations like frozen, freeze-dried, irradiated, or demineralized ${ }^{7}$. Transplantation of allogenic bone as a method of treatment as various disorders of skeleton was started late decade of $19^{\text {th }}$ century and $1^{\text {st }}$ decade of $20^{\text {th }}$ century. Freeze-dried bone allograft was first introduced by United State Navy tissue Bank.

It has been observed that 177 solitary bone cyst have been treated with curettage and packing with freeze dried cancellous bone allograft, where 97(55.0\%) cases are completely healed; 64(34\%) cases are recurred and $16(9 \%)$ cases have a non-progressive residual defect that did not require an operation; in addition the overall recurrence rate was $45.0 \%$ cases $^{7}$. Though allografts are attractive sources, there are several problems encountered in using them, including resorbtion, relative risk of disease transmission, immunogenicity, loss of biologic and mechanical properties secondary to its processing, and non-availability world-wide due to financial and religious concerns ${ }^{8}$.

Scintigraphic images are obtained utilizing the intravenous administration of a radiopharmaceutical, particularly technetium- $99 \mathrm{~m}$ labeled diphosphonates. In an in vitro binding assay, the competitive adsorption of technetium- $99 \mathrm{~m}$ labeled diphosphonates to pure hydroxyapatite is forty times greater than to pure organic bone matrix. Thus, its uptake correlates well with the rate of mineralization". Harbert $^{10}$ has mentioned that bone scintigraphy provides a means of predicting graft failure before radiographic or clinical changes become apparent and, thus, helping to avoid a loss of surrounding bone from graft necrosis or infection.

Thus the study was designed to evaluate the integration of HA and allogenic bone as bone graft by assessment of histopathological evaluation and scintigraphic assessment of the graft to its future use with maxillofacial reconstruction.

\section{Methodology}

Study Population and Settings: This single centre, parallel, randomized clinical trial was conducted in the Department of Oral and Maxillofacial Surgery at Bangabandhu Sheikh Mujib Medical University, Dhaka, Bangladesh from July 2006 to June 2008 for a period of two (02) years. Patients presented with radicular and dentigerous cyst with the size of the lesion between $2 \mathrm{~cm}$ to $7 \mathrm{~cm}$. in diameter and relatively non infected cystic lesion were selected as study population. Patients with any systemic bone disease or patients with comorbidities like diabetes mellitus, tuberculosis, rheumatic heart diseases and renal failure patients or patients who had taken radiotherapy in the orofacial region were excluded from this study. Written informed consent was taken from the patient or legal guardian. Permission for the study was taken from the concerned department of BSMMU.

Randomization and Blinding: Patients were included in the study after the clinical and radiological evaluation. The computer generated simple random sampling technique was applied to divide the study population into two groups.

Allocation: The half of total cases were treated with allogenic-bone graft and rest half cases were treated hydroxyapatite alloplastic material after enucleation of the cystic lesion. Medical grade Hydroxyapatite was prepared according to manufacturer instructions. The powders was sterilized by applying a Co60 gamma-ray radiation at the dosage of $2.5 \mathrm{MRad}$. 
Lyophilized radiation sterilized bone allograft supplied by Tissue Bank and Biomaterial Research unit, Atomic Energy Research Establishment, Savar, Institute of Food and radiation biology, Bangladesh Atomic Energy Commission, Savar, Dhaka. Sterilization of tissues by $60 \mathrm{CO}$ Gamma Radiation to destroy or inactivate or reduces the microorganism to a Sterility Assurance Level (SAL) of 10-6. Preoperatively routine blood test, random blood sugar (RBS), serum urea, creatinine, serum alkaline phosphatase, serum calcium level, and radiological examination in all cases were done.

Surgical procedure: The standard surgical procedure was to be performed meticulously in sterile environment under local anesthesia and sedation. Enucleation of cyst was done with removal of all granulation tissue. After enucleation of cyst bony cavity was irrigated with diluted povidone iodine for the purpose of debridment. Granules of hydroxyapatite or allobone was placed on the surgical defect and wetted with patient blood or saline solution followed by primary closure of the wound. Prophylactic antibiotics like Cephradine $500 \mathrm{mg} 8$ hourly continued for 7 days, Metronidazole $400 \mathrm{mg}$ three times daily for 5-7 days, analgesics like NSAID and mouthwash was prescribed.

Follow Up and Outcome Measures: The wound was checked on the following day and radiograph was done three days after operation. Sutures were removed on seventh post-operative day. Post-operative radiograph were taken after one month, three months and six months. Radiographs for each patient was done from the same diagnostic center with the same machine and the operator. Then the Initial diameter of the lesion was measured and follow-up was done for each patient with occlusal view or OPG or intraoral periapical view with the same magnification, exposure time in the same $\mathrm{X}$ ray machine with the same operator. Each OPG was done by Cranex Base X (Finland) panoramic dental X-ray machine with $\mathrm{mA}$ set to 10 and $\mathrm{KV}$ set to 75 for each patient. The margin of the successful bone grafts showed less sharpness than the immediate post-operative margin in each case after one month. Trabecular pattern of woven bone with each patient was evaluated visually by comparing the X-ray of the same patient from two different focuses using a standard scale with image processing software. Histopathological examination was done after six month of operation. Tissue specimen was taken from all these cases after reflecting the mucoperiosteum at the previous lesion area to find out the presence or absence of cementing line, osteoblast, woven bone, fibrous and chronic inflammatory cell to assess the quality of the graft material to the healed lesion. This was done in the department of Pathology, BSMMU with their valuable pathological report. Scintigraphic examination was performed after 6 months of the operation to evaluate the viability of the bone graft in the graft site. Three phase scan was done with technetium- 99m labeled diphosphonates for each patient to access the viability of bone graft or areas of increased bone metabolism were evidence of good viability and was appeared as areas of increased radiotracer uptake, namely "hot spots. Diminished or absent uptake, are called "cold spots."

Statistical Analysis: All the data sheet of history and $\mathrm{x}$-ray were collected and were analyzed scientifically. Computer based statistical analysis was carried out with appropriate techniques and systems. Results were presented as a detailed pathology report that includes high-resolution digital photomicrographs with image processing software. Result of study was calculated by standard statistical method. To provide a correct and unbiased result of the study, consultation was taken with an expert bio-statistician. Chi-square test was done to find out association between different variables and ' $t$ ' test was done for comparing outcome scores between the treatment groups. Level of significance was set at $\mathrm{P}<0.05$.

\section{Results}

The main objective of this study was to find out the rate of infection for the patient with use of hydroxyapatite alloplastic material and allogenic bone graft. A total number of 28 patients were recruited for this study of which 14 patients were treated with hydroxyapatite crystals bone graft in group A and 14 patients were treated with allogenic bone graft in group B. Follow-up data were collected after 1, 3 and 6 months of bone graft. The age of patients of both groups ranged between 14 and 58 years. In group A, the highest number of patients was in the age group 31 to 40 years; and in group $\mathrm{B}$, the highest number of patients was in the age group of 21 to 30 years (Table 1).

In histopathological examination marked presence of cementing line, woven bone and osteoblast cell was found in patients who were grafted with hydroxyaptite and this had indicated good osteoblastic activity. On the other hand marked presence of chronic inflammatory cell and fibrous tissue in allo-bone 
grafting patient had indicated less osteoblastic activity (Table 2).

Table 1: Distribution of patients of both groups by age

\begin{tabular}{lcc}
\hline Age Group & Group A & Group B \\
\hline Up to 20 Years & $4(28.6 \%)$ & $4(28.6 \%)$ \\
21 to 30 Years & $3(21.4 \%)$ & $7(50.0 \%)$ \\
31 to 40 Years & $5(35.7 \%)$ & $2(14.3 \%)$ \\
More than 40 Years & $2(14.3 \%)$ & $1(7.1 \%)$ \\
Total & $\mathbf{1 4 ( 1 0 0 . 0 \% )}$ & $\mathbf{1 4 ( 1 0 0 . 0 \% )}$ \\
\hline
\end{tabular}

Group-A: With Hydroxyapatite bone graft; Group-B: With Allogenic bone graft

Table 2: Comparison of Histopathological Outcomes of the Patients between Group A and Group B

\begin{tabular}{lcc}
\hline Histopathological Outcomes & Group A & Group B \\
\hline Cementing line & ++ & + \\
Woven bone & ++ & + \\
Osteoblast cell & ++ & + \\
Chronic Inflammatory cell & - & ++ \\
Fibrous tissue & - & ++ \\
\hline
\end{tabular}

'+' Indicates present; '- ' Indicates absent

Increased technetium-99m labeled diphosphonate uptake was found in the patient with allograft which had indicated good osteoblastic activity and good viability of the graft. Lightly increased uptake had indicated the chronic inflammation and fibrous healing in the allogenic bone graft group indicates average healing of the graft (Table 3).

Table 3: Comparison of Outcomes of the Patients between Two Management Groups In Relation To Scintigram with Hydroxapatite and With Allograft

\begin{tabular}{lcc}
\hline Outcomes & Group A & Group B \\
\hline Diphosphonate uptake & ++ & + \\
Healing quality & Good & Average \\
\hline
\end{tabular}

Diphosphonate uptake=Technetium-99m labeled diphosphonate uptake; '+ +' Indicates good tracer uptake; ' +' Indicates slightly increased tracer uptake

In the summary of the major observations, there was a smaller lesion diameter of hydroxyapatite group in comparison with allogenic bone graft group which had indicated comparatively better healing than that of the hydroxyapatite group. In observation of trabecular pattern, histological outcome and scintigraphic outcome the hyroxyapaite group showed better quality of healing than that of allogenic bone graft group (Table 4).
Table 4: Summary of Different Observations between Two Groups

\begin{tabular}{lcc}
\hline Major Observation & Group A & Group B \\
\hline Lesion diameter & Less & More \\
Trabecular pattern & Good & Average \\
Histological examination & Good & Average \\
Scintigraphic examination & Good & Average \\
\hline
\end{tabular}

\section{Discussion}

Multitudes of materials were used as bone graft in maxillofacial region ${ }^{11}$. This present study is undertaken to compare the outcomes of allogenic bone and hydroxyapatite bone graft in cystic cavities after enucleation. The radiological and histological changes of the bone grafts are assessed to determine the true nature and quality of healing to use it further in our field. Scintigraphy is used as a marker to determine the viability of the grafts.

In this study 28 patients were included after the clinical and radiological evaluation where 14 cases were treated with allogenic-bone graft and the rest 14 cases were treated with hydroxyapatite alloplastic-bone graft material after enucleation of the cystic lesion.

It has been found that hydroxyapatite bone graft is both clinically and radiologically better than allogenic bone graft in reducing the diameter of lesion. It has been supported by Zuev et $\mathrm{al}^{12}$. This observation has been concluded that the patients with hydroxapatite bone graft has showed better radiological outcome than their allogenic counterparts. They have also revealed the outcomes of two groups which is poorly defined in the initial few weeks; however, in the later period like three months it is significant and this result is very close to Schwartz et a $1^{13}$.

In this study patients with hydroxapatite bone graft showed superior trabecular pattern than the allogenic counterparts. The difference of trabecular pattern between the two groups started to amplify in the post-operative period. The difference was almost persistent throughout the period of 6 months, with gradual increasing tendency.

Histopathological slide has been prepared from cases of both groups of same site and procedure after six months of operation. According to the histopathological findings the neo-osteogenetic process is better in the hydroxyapatite group. Microscopic findings have revealed that the graft is seated in the duel process of osteoclastic and osteoblastic activities with the ingrowths of capillaries. There is a marked cementing line which indicates deposition of woven bone, on the other hand there is less definite 
histological feature of active bone formation rather fibrous deposition showed in allogenic bone group. Moreover the infiltration of chronic inflammatory cell is evident in allo group. This indicates that the hydroxyapatite group is better than allogenic bone group and this result is consistent with the other studies $^{14-16}$.

Radionuclide bone scanning has been done from randomly selected cases of both groups. More increased tracer uptake is seen in the operation site of hydroxyapatite group than in the allo group which is also supported by the finding. More tracer uptake of radioactive iodine indicates good osteoblastic activity which is supported by other studies ${ }^{17-18}$. On the other hand slight tracer uptake indicates average or poor osteoblastic activity.

Zanetti and Nassif ${ }^{19}$ presented their initial experience with the use of flexible hydroxyapatite sheets to repair lateral skull base defects. They prospectively reviewed the outcomes of seven patients with mastoid defects, 3 $\mathrm{cm}$ or less, resulting from traumatic, iatrogenic, or infectious causes. After a mean follow-up of 27.5 months, none of the patients suffered any complications related to the graft such as infection, extrusion, or cerebrospinal fluid leaks. All patients exhibited a reasonable anatomic contour on postoperative computed tomographic scans. Although this experience is limited, flexible, osteoconductive hydroxyapatite sheets offer a viable alternative for the repair of skull base defects, and further clinical evaluation is warranted.

Shields et $\mathrm{al}^{20}$ reported their experience with 250 consecutive cases of hydroxyapatite orbital implant placed after enucleation with minimum complications and with no cases of orbital hemorrhage, implant migration,or implant extrusion.

\section{Conclusion}

In conclusion the radiological, histopathological and scintigraphical outcome of the patients who were treated with hydroxyaptite granule bone graft were satisfactory in comparison with those who were treated with allogenic bone graft. There is a smaller lesion diameter of hydroxyapatite group in comparison with allogenic bone graft group. This indicates comparatively better healing than that of the allogenic bone graft group. In observation of trabecular pattern, histological outcome and scintigraphic outcome of the hyroxyapaite group shows better quality of healing than that of allogenic bone graft group. Large scale study should be conducted to get the real scenario.

\section{References}

1. Chiapasco M, Rossi A, Motta JJ, Crescentini M. Spontaneous bone regeneration after enucleation of large mandibular cysts: a radiographic computed analysis of 27 consecutive cases. Journal of oral and maxillofacial surgery. 2000 Sep 1;58(9):942-8

2. Bodner L. Osseous regeneration in the jaws using demineralized allogenic bone implants. Journal of Cranio-Maxillofacial Surgery. 1998 Apr 1;26(2):116-20.

3. Bohner M. Calcium orthophosphates in medicine: from ceramics to calcium phosphate cements. Injury. 2000 Dec 1;31:D37-47.

4. Stoelinga PJW, Bronkhorst FB. The incidence, multiple presentation and recurrence of aggressive cysts of the jaws. J Craniomaxillofac Surg. 1988;16:184-195

5. Pradel W, Eckelt U, Lauer G. Bone regeneration after enucleation of mandibular cysts: comparing autogenous grafts from tissue-engineered bone and iliac bone. Oral Surgery, Oral Medicine, Oral Pathology, Oral Radiology, and Endodontology. 2006 Mar 1;101(3):285-90

6. Ettl T, Gosau M, Sader R, Reichert TE. Jaw cysts-Filling or no filling after enucleation? A review. Journal of Cranio-Maxillofacial Surgery. 2012 Sep 1;40(6):485-93.

7. Thorwarth M, Schultze-Mosgau S, Kessler P, Wiltfang J, Schlegel KA. Bone regeneration in osseous defects using a resorbable nanoparticular hydroxyapatite. Journal of oral and maxillofacial surgery. 2005 Nov 1;63(11):1626-33.

8. Bodner L. Cystic lesions of the jaws in children. International journal of pediatric otorhinolaryngology. 2002 Jan 11;62(1):25-9.

9. Francis MD, Horn PA, Tofe AJ. Controversial mechanism of technetium-99m deposition on bone. InJournal of Nuclear Medicine 1981 Jan 1 (Vol. 22, No. 6, pp. P72-P72). 1850 SAMUEL MORSE DR, RESTON, VA 20190-5316: SOC NUCLEAR MEDICINE INC.

10. Harbert JC 1996, 'The musculoeskeletal system', in: Harbert JC, Eckelman WC, Newmann RD (eds), Nuclear medicine: diagnosis and therapy. Thieme, New York, pp 801-63

11. Shear M, Speight PM. Cysts of the oral and maxillofacial regions. Munksgaard: Blackwell; 2007

12. Zuev VP, Dmitrieva LA, Pankratov AS, Filatova NA. The comparative characteristics of stimulators of reparative osteogenesis in the treatment of periodontal diseases. Stomatologiia. 1996;75(5):31-4.

13. Schwartz Z, Mellonig JT, Carnes Jr DL, De La Fontaine J, Cochran DL, Dean DD, Boyan BD. Ability of commercial demineralized freeze $\square$ dried bone allograft to induce new bone formation. Journal of periodontology. 1996 Sep;67(9):918-26.

14. Philipsen HP. Keratocystic odontogenic tumor. In: Barnes L, Eveson JW, Reichart P, Sidransky D, editors. Head and neck tumours, WHO classification of tumours. Lyon: IARC Press; 2005. pp. 306-307

15. Kuttenberger JJ, Farmand M, Sto H. Recurrence of a solitary bone cyst of the mandibular condyle in a bone graft: a case report. Oral surgery, oral medicine, oral pathology. 1992 Nov 1;74(5):550-6

16. Lundgren S, Andersson S, Sennerby L. Spontaneous bone formation in the maxillary sinus after removal of a cyst: coincidence or consequence?. Clinical implant dentistry and related research. 2003 Aug;5(2):78-81.

17. Lockhart R, Ceccaldi J, Bertrand JC. Postoperative maxillary cyst following sinus bone graft: report of a case. International Journal of Oral \& Maxillofacial Implants. 2000 Jul 1;15(4).

18. Bezrukov VM, Grigor'iants LA, Zuev VP, Pankratov AS. The surgical treatment of jaw cysts using hydroxyapatite with an ultrahigh degree of dispersity. Stomatologiia. 1998;77(1):31-5. 
19. Zanetti D, Nassif N. Transmastoid repair of minor skull base defects with flexible hydroxyapatite sheets. Skull Base. 2003;13(01):001-12.

20. Shields CL, Shields JA, De Potter P, Singh AD. Lack of complications of the hydroxyapatite orbital implant in 250 consecutive cases. Transactions of the American Ophthalmological Society. 1993;91:177 